\title{
ON NEAR-RING IDEMPOTENTS AND POLYNOMIALS ON DIRECT PRODUCTS OF $\Omega$-GROUPS
}

\author{
ERHARD AICHINGER \\ Institut für Algebra, Stochastik und wissensbasierte mathematische Systeme, \\ J. Kepler Univ., Linz, 4040 Linz, Austria (erhard@algebra.uni-linz.ac.at)
}

(Received 23 December 1999)

\begin{abstract}
Let $N$ be a zero-symmetric near-ring with identity, and let $\Gamma$ be a faithful tame $N$-group. We characterize those ideals of $\Gamma$ that are the range of some idempotent element of $N$. Using these idempotents, we show that the polynomials on the direct product of the finite $\Omega$-groups $V_{1}, V_{2}, \ldots, V_{n}$ can be studied componentwise if and only if $\prod_{i=1}^{n} V_{i}$ has no skew congruences.
\end{abstract}

Keywords: near-rings; $\Omega$-groups; polynomials; $N$-groups

AMS 2000 Mathematics subject classification: Primary 16Y30

Secondary 08A40

\section{Ideals that are the range of a near-ring element}

For the basic notions of near-ring theory, such as near-ring, zero-symmetric, left ideal, $N$-group, ideal, we refer to [13].

Let $N$ be a zero-symmetric near-ring with identity id. Then the unital $N$-group $\Gamma$ is called tame [17] if and only if for all $\gamma, x \in \Gamma$ and $\boldsymbol{n} \in N$ there is an element $\boldsymbol{m} \in N$ such that

$$
\boldsymbol{n} \cdot(\gamma+x)-\boldsymbol{n} \cdot \gamma=\boldsymbol{m} \cdot x
$$

We write near-ring elements in bold italic and $N$-group elements in plain italic. Every $N$-subgroup of a tame $N$-group $\Gamma$ is an ideal of $\Gamma$. The lattice of ideals of $\Gamma$ will be abbreviated by $\mathrm{Id} \Gamma$; the sum of the ideals $I$ and $J$ is written as $I \vee J$, their intersection as $I \wedge J$. It is well known that for $A, B \in \operatorname{Id} \Gamma, a \in A, b \in B, \boldsymbol{n} \in N$, the element $\boldsymbol{n} \cdot a+\boldsymbol{n} \cdot b-\boldsymbol{n} \cdot(a+b)$ lies in $A \wedge B$ [16, Proposition 1.3]. For two ideals $A, B \in \operatorname{ld} \Gamma$ with $A \leqslant B$, we denote the interval $\{I \in \operatorname{ld} \Gamma \mid A \leqslant I \leqslant B\}$ by $I[A, B]$. We write $A \prec B$ ( $A$ is a subcover of $B$ ) if and only if $A<B$ and there is no $I \in \operatorname{Id} \Gamma$ with $A<I<B$. We call an ideal $A$ of $\Gamma$ join irreducible if and only if $A=B \vee C$ implies $A=B$ or $A=C$. In a lattice with both chain conditions, the join irreducible element $A$ has a unique subcover; we abbreviate it by $A^{-}$. We say that two $N$-groups $G$ and $H$ are $N$-isomorphic if and only if there is a group isomorphism $\varphi: G \rightarrow H$ with $\boldsymbol{n} \cdot \varphi(g)=\varphi(\boldsymbol{n} \cdot g)$ for all $\boldsymbol{n} \in N$, $g \in G$. For $A, B$ in Id $\Gamma$ with $A \leqslant B$, we define the set $B / A:=\{b+A \mid b \in B\}$. On this 
set we define addition by $\left(b_{1}+A\right)+\left(b_{2}+A\right):=\left(b_{1}+b_{2}\right)+A$, and the operation of $N$ by $\boldsymbol{n} \cdot(b+A):=(\boldsymbol{n} \cdot b)+A$. If $\Gamma$ is a tame $N$-group and $A, B$ are ideals of $\Gamma$ with $A \leqslant B$, then the $N$-group $B / A$ is tame, too. If, furthermore, $A \prec B$ in Id $\Gamma$, then the $N$-group $B / A$ has no ideals except $0=A / A$ and $B / A$. We write $\operatorname{Ann}(B / A)$ for the annihilator of $B / A$, which means

$$
\operatorname{Ann}(B / A)=\{\boldsymbol{n} \in N \mid \boldsymbol{n} \cdot B \subseteq A\} .
$$

The annihilator $\operatorname{Ann}(B / A)$ is an ideal of the near-ring $(N,+, \circ)$. If the tame near-ring $N$ has the descending chain condition (DCC) on left ideals (called DCCL from now on), and if $A \prec B$ in Id $\Gamma$, the near-ring $N / \operatorname{Ann}(B / A)$ is 2-primitive. A 2-primitive near-ring $R$ with identity and DCCL is either the full matrix-ring of $k \times k$-matrices over a division ring, in which case it is simple [8, Theorem (3.1), p. 32], or, if it is not a ring, then it is a centralizer near-ring. By $[\mathbf{3}, \mathbf{1 2}]$, every 2-primitive near-ring with identity and DCCL which is not a ring is simple. Altogether, $N / \operatorname{Ann}(B / A)$ is simple, and thus $\operatorname{Ann}(B / A)$ is a maximal ideal of $N$.

Our first result characterizes those $N$-subgroups of $\Gamma$ that are the range of an idempotent element of $N$.

Theorem 1.1. Let $N$ be a zero-symmetric near-ring with identity, and let $\Gamma$ be a tame $N$-group. We assume that $N$ has the DCC on left ideals, and that the ideal lattice of $\Gamma$ satisfies both the ascending chain condition (ACC) and the DCC. Let $H$ be an $N$-subgroup of $\Gamma$. Then the following are equivalent.

(1) There is an element $\boldsymbol{e} \in N$ with $\boldsymbol{e} \cdot h=h$ for all $h \in H$, and $\boldsymbol{e} \cdot \Gamma \subseteq H$.

(2) If $A$ and $D$ are join irreducible ideals in $\operatorname{ld} \Gamma$, and if $A \leqslant H$ and the $N$-groups $A / A^{-}$and $D / D^{-}$are $N$-isomorphic, then $D \leqslant H$.

Proof. (1) $\Rightarrow(2)$. We fix two join irreducible ideals $A, B \in \operatorname{ld} \Gamma$ with $A \leqslant H$ such that $A / A^{-}$and $B / B^{-}$are $N$-isomorphic. We have $(\boldsymbol{e}-\mathbf{i d}) \cdot a=0$ for all $a \in A$, and therefore $(\boldsymbol{e}-\mathbf{i d}) \cdot\left(a+A^{-}\right)=0+A^{-}$for all $a \in A$. Since $A / A^{-}$and $B / B^{-}$are $N$-isomorphic, $(\boldsymbol{e}-\mathbf{i d}) \cdot\left(b+B^{-}\right)=0+B^{-}$for all $b \in B$. Thus for every $b \in B, b$ is congruent to $e \cdot b$ modulo $B^{-}$. Since $\boldsymbol{e} \cdot b$ lies in $H$, we get

$$
\text { for all } b \in B: b \in B^{-} \vee H \text {. }
$$

Hence $B \leqslant B^{-} \vee H$, and therefore $B=B \wedge\left(B^{-} \vee H\right)$, and, by the modularity of the lattice Id $\Gamma, B=B^{-} \vee(B \wedge H)$. Since $B$ is join irreducible, we obtain $B \wedge H=B$, and therefore $B \leqslant H$. This completes the proof of $(1) \Rightarrow(2)$.

(2) $\Rightarrow(1)$. We may assume $H \neq 0$. We define a subset $\mathcal{B}$ of $\operatorname{Id} \Gamma$ by

$$
\mathcal{B}:=\{B \in \operatorname{ld} \Gamma \mid \text { there is no } \boldsymbol{p} \in N \text { with }(\boldsymbol{p}-\mathbf{i d}) \cdot H=0 \text { and } \boldsymbol{p} \cdot B \subseteq H\} .
$$

Our endeavour will be to show $\mathcal{B}=\emptyset$, because then $\Gamma \notin \mathcal{B}$, which yields a $\boldsymbol{p} \in N$ with $\boldsymbol{p} \cdot h=h$ for all $h \in H$, and $\boldsymbol{p} \cdot \Gamma \subseteq H$. Seeking a contradiction, we suppose $\mathcal{B} \neq \emptyset$. Since Id $\Gamma$ has the DCC, $\mathcal{B}$ has a minimal element, say $D$. Since $0 \notin \mathcal{B}$ (take $\boldsymbol{p}:=\mathbf{i d}$ in (1.1)), we have $D \neq 0$. We will now show that

$$
D \text { is join irreducible. }
$$


Suppose $D=D_{1} \vee D_{2}$ with $D_{1}<D, D_{2}<D$. By the minimality of $D$, we have $D_{1} \notin \mathcal{B}$, $D_{2} \notin \mathcal{B}$. Hence there are $\boldsymbol{p}_{1}, \boldsymbol{p}_{2} \in N$ with

$$
\begin{aligned}
& \boldsymbol{p}_{1} \cdot D_{1} \subseteq H, \quad \forall h \in H: \boldsymbol{p}_{1} \cdot h=h, \\
& \boldsymbol{p}_{2} \cdot D_{2} \subseteq H, \quad \forall h \in H: \boldsymbol{p}_{2} \cdot h=h .
\end{aligned}
$$

We define $\boldsymbol{p}_{3}:=\boldsymbol{p}_{2} \circ \boldsymbol{p}_{1}$. We have $\boldsymbol{p}_{3} \cdot D_{1} \subseteq H$ and $\boldsymbol{p}_{3} \cdot D_{2} \subseteq H$. Let $\boldsymbol{p}_{4}:=\boldsymbol{p}_{3} \circ \boldsymbol{p}_{3}$. We show

$$
\boldsymbol{p}_{4} \cdot D \subseteq H .
$$

We fix $d \in D$. Since $D_{1} \vee D_{2}=D$, there are $d_{1} \in D_{1}, d_{2} \in D_{2}$ with $d=d_{1}+d_{2}$. We first compute $\boldsymbol{p}_{3} \cdot\left(d_{1}+d_{2}\right)$. Since $\boldsymbol{p}_{3} \cdot\left(d_{1}+d_{2}\right)$ is congruent to $\boldsymbol{p}_{3} \cdot d_{1}+\boldsymbol{p}_{3} \cdot d_{2}$ modulo $D_{1} \wedge D_{2}$, there is an element $d_{3} \in D_{1} \wedge D_{2}$ such that

$$
\boldsymbol{p}_{3} \cdot\left(d_{1}+d_{2}\right)=\left(\boldsymbol{p}_{3} \cdot d_{1}+\boldsymbol{p}_{3} \cdot d_{2}\right)+d_{3} .
$$

Since $\boldsymbol{p}_{3} \cdot d_{1} \in H$ and $\boldsymbol{p}_{3} \cdot d_{2} \in H$, there is an element $h \in H$ such that $\boldsymbol{p}_{3} \cdot\left(d_{1}+d_{2}\right)=h+d_{3}$. Applying $\boldsymbol{p}_{3}$ again, we obtain

$$
\begin{aligned}
\left(\boldsymbol{p}_{3} \circ \boldsymbol{p}_{3}\right) \cdot\left(d_{1}+d_{2}\right) & =\boldsymbol{p}_{3} \cdot\left(h+d_{3}\right) \\
& =\left(\boldsymbol{p}_{3} \cdot h+\boldsymbol{p}_{3} \cdot d_{3}\right)+d_{4},
\end{aligned}
$$

where $d_{4}$ lies in $H \wedge\left(D_{1} \wedge D_{2}\right)$. Therefore every summand of $\boldsymbol{p}_{3} \cdot h+\boldsymbol{p}_{3} \cdot d_{3}+d_{4}$ lies in $H$, which proves (1.3). Altogether, we have $\boldsymbol{p}_{4} \cdot h=h$ for all $h \in H$ and $\boldsymbol{p}_{4} \cdot D \subseteq H$. This shows that $D$ is not in $\mathcal{B}$, a contradiction to the choice of $D$, and completes the proof of (1.2).

The next step is to show that

$$
\text { for all } \boldsymbol{p} \in N: \boldsymbol{p} \cdot H=0 \Rightarrow \boldsymbol{p} \cdot D \subseteq D^{-} .
$$

We consider the ideals $\operatorname{Ann}(H)=\{\boldsymbol{n} \in N \mid \boldsymbol{n} \cdot H=0\}$ and $\operatorname{Ann}\left(D / D^{-}\right)=\{\boldsymbol{n} \in$ $\left.N \mid \boldsymbol{n} \cdot D \subseteq D^{-}\right\}$. Since $D^{-} \prec D$ in $\operatorname{ld} \Gamma$, the $N$-group $D / D^{-}$has no proper $N$ subgroups; therefore $N / \operatorname{Ann}\left(D / D^{-}\right)$is 2-primitive on $\operatorname{Ann}\left(D / D^{-}\right)$, and thus simple. Hence $\operatorname{Ann}\left(D / D^{-}\right)$is a maximal ideal of $N$. Supposing that (1.4) fails, we have $\operatorname{Ann}(H) \nless$ $\operatorname{Ann}\left(D / D^{-}\right)$. By the maximality of $\operatorname{Ann}\left(D / D^{-}\right)$, this implies

$$
\operatorname{Ann}(H)+\operatorname{Ann}\left(D / D^{-}\right)=N .
$$

Thus there are $\boldsymbol{a} \in \operatorname{Ann}(H), \boldsymbol{i} \in \operatorname{Ann}\left(D / D^{-}\right)$with $\boldsymbol{a}+\boldsymbol{i}=\mathbf{i d}$. The element $\boldsymbol{i}$ satisfies $\boldsymbol{i} \cdot D \subseteq D^{-}$and $\boldsymbol{i} \cdot h=(-\boldsymbol{a}+\mathbf{i d}) \cdot h=-0+h=h$ for all $h \in H$. Since $D$ is minimal in $\mathcal{B}$, we have $D^{-} \notin \mathcal{B}$. So there exists $\boldsymbol{p}_{5} \in N$ with $\boldsymbol{p}_{5} \cdot h=h$ for all $h \in H$ and $\boldsymbol{p}_{5} \cdot D^{-} \subseteq H$. We consider the element $\boldsymbol{p}_{6} \in N$ defined by $\boldsymbol{p}_{6}:=\boldsymbol{p}_{5} \circ \boldsymbol{i}$. It is easy to see that $\boldsymbol{p}_{6} \cdot h=h$ for all $h \in H$. We also have $\boldsymbol{p}_{6} \cdot D=\boldsymbol{p}_{5} \cdot(\boldsymbol{i} \cdot D) \subseteq \boldsymbol{p}_{5} \cdot D^{-} \subseteq H$. The existence of such a $\boldsymbol{p}_{6} \in N$ implies $D \notin \mathcal{B}$; this contradiction proves (1.4). We defer the rest of the proof of Theorem 1.1 to p. 384 because we first need some facts about near-rings. 
Let $N$ be a near-ring which is tame on $\Gamma$. In the sequel, we state two conditions on ideals $A, B, C, D$ with $A \leqslant B$ and $C \leqslant D$ that imply that the $N$-groups $B / A$ and $D / C$ are $N$-isomorphic. We express the first condition using notions from lattice theory. For $A, B, C, D \in \operatorname{Id} \Gamma$ with $A \leqslant B, C \leqslant D$, we say that the interval $I[A, B]$ projects up to $I[C, D]$ if and only if $A=B \wedge C$ and $D=B \vee C$, and we write $I[A, B] \nearrow I[C, D]$ or $I[C, D] \searrow I[A, B]$. The smallest equivalence relation that contains $\nearrow$ will be abbreviated by $\rightsquigarrow$. If $I[A, B] \leadsto I[C, D]$, we say that the two intervals are projective.

Lemma 1.2 (cf. [1]). Let $N$ be a zero-symmetric near-ring, let $\Gamma$ be a tame $N$-group, and let $A, B, C, D$ be ideals of $\Gamma$ with $A \leqslant B, C \leqslant D$ such that the intervals $I[A, B]$ and $I[C, D]$ are projective. Then the $N$-groups $B / A$ and $D / C$ are $N$-isomorphic.

Proof. We assume $I[A, B] \nearrow I[C, D]$. Then every element in $d \in D$ can be written as $d=b+c$ with $b \in B, c \in C$. The mapping $\varphi: D / C \rightarrow B / A$ with $\varphi((b+c)+C)=b+A$ is an isomorphism.

The result is actually well-known as the homomorphism theorem $(B+C) / B \cong_{N}$ $B /(C \cap B)$ [11, Theorem 2.28].

The next method to find isomorphic $N$-groups is a generalization of the known fact that for a finite simple ring with unit $R$, all faithful simple unital $R$-modules are isomorphic (cf. [14, Proposition 2.1.15, p. 154], [3, Theorem 4.3]). We need the following lemma.

Lemma 1.3 (cf. Proposition 2.2 of [1], [2] and Theorem 4.56(a) of [13]). Let $N$ be a zero-symmetric near-ring, let $I$ be an ideal of $N$, and let $\Gamma$ be an $N$-group that satisfies $\operatorname{Ann}(\Gamma)=I$ and $N \cdot \gamma=\Gamma$ for all $\gamma \in \Gamma$ with $\gamma \neq 0$. We assume that we have a left ideal $L$ of $N$ such that $L>I$ and there is no left ideal $L^{\prime}$ of $N$ with $L>L^{\prime}>I$. Then the $N$-group $\Gamma$ is $N$-isomorphic to the $N$-group $L / I$.

Proof. Since $L \notin \operatorname{Ann}(\Gamma)$, we have elements $\boldsymbol{l}_{0} \in L, \gamma_{0} \in \Gamma$ with $\boldsymbol{l}_{0} \cdot \gamma_{0} \neq 0$. We define a mapping $\varphi$ by

$$
\varphi: L \rightarrow \Gamma, \quad \boldsymbol{l} \mapsto \boldsymbol{l} \cdot \gamma_{0} .
$$

It is easy to see that $\varphi$ is an $N$-homomorphism from the $N$-group $L$ to the $N$-group $\Gamma$. Since $\boldsymbol{l}_{0} \cdot \gamma_{0} \neq 0$, the assumptions on $\Gamma$ yield $N \cdot \boldsymbol{l}_{0} \cdot \gamma_{0}=\Gamma$. Since $N \cdot \boldsymbol{l}_{0} \subseteq L$, we get $L \cdot \gamma_{0}=\Gamma$, and hence $\varphi$ is surjective. We take $L^{\prime}$ to be the kernel of $\varphi$, i.e.

$$
L^{\prime}=\left\{\boldsymbol{l} \in L \mid \boldsymbol{l} \cdot \gamma_{0}=0\right\} .
$$

We check that $L^{\prime}$ is a left ideal of $N$. Furthermore, every element of $I=\operatorname{Ann}(\Gamma)$ lies in $L^{\prime}$. So we have

$$
I \leqslant L^{\prime} \leqslant L .
$$

Since by the assumptions $L$ covers $I, L^{\prime}$ has to be either $L$ or $I$. The element $l_{0}$ shows $L^{\prime}<L$, and so $L^{\prime}=I$. The homomorphism theorem yields that the $N$-group $L / \operatorname{ker} \varphi=$ $L / I$ is $N$-isomorphic to $\Gamma$.

If two $N$-groups are $N$-isomorphic, they have the same annihilators. Sometimes, the converse is true. 
Corollary 1.4 (cf. [1]). Let $N$ be a zero-symmetric near-ring with identity, let $\Gamma$ be a tame $N$-group, and let $A, B, C, D$ be ideals of $\Gamma$ with $A \prec B, C \prec D$, and $\operatorname{Ann}(B / A)=\operatorname{Ann}(D / C)$. If the near-ring $N / \operatorname{Ann}(B / A)$ has a minimal left ideal, the $N$-groups $B / A$ and $D / C$ are $N$-isomorphic.

Proof. Since $A \prec B$, and since $\Gamma$ is a tame $N$-group, $B / A$ has no $N$-subgroups. For every $\beta \in B / A$, the set $N \cdot \beta$ forms an $N$-subgroup of $B / A$, and since for the identity of id of $N$ we have id $\beta=\beta$, we see $N \cdot \beta=B / A$ for every non-zero $\beta \in B / A$. The fact that $N / \operatorname{Ann}(B / A)$ has a minimal left ideal gives us the left ideal $L$ required in the assumptions of Lemma 1.3. Now the result follows from this Lemma.

Lemma 1.5 (cf. [2]). Let $N$ be a zero-symmetric near-ring with identity and the $D C C$ on left ideals, let $\Gamma$ be a tame $N$-group such that Id $\Gamma$ satisfies both the ACC and the $D C C$, and let $A, B, C, D \in \operatorname{Id} \Gamma$ such that $C \leqslant D, A \prec B$. We assume that each $\boldsymbol{p} \in N$ with $\boldsymbol{p} \cdot D \subseteq C$ satisfies $\boldsymbol{p} \cdot B \subseteq A$. Then there are ideals $C^{\prime}, D^{\prime}$ in Id $\Gamma$ with $C \leqslant C^{\prime} \prec D^{\prime} \leqslant D$ such that $D^{\prime} / C^{\prime}$ and $B / A$ are $N$-isomorphic.

Proof. We take $I$ to be the ideal $\operatorname{Ann}(B / A)$ of $N$. Since $A \prec B$ in Id $\Gamma$, the near-ring $N / I$ is 2-primitive on $B / A$, and thus, as a 2-primitive near-ring with DCCL, simple. Hence $I$ is a maximal ideal of $N$.

Let $C_{1}$ be any ideal of $\Gamma$ in $I[C, D]$. Let $J_{1}, J_{2}$ be the ideals of $N$ defined by

$$
J_{1}:=\operatorname{Ann}\left(C_{1} / C\right), \quad J_{2}:=\operatorname{Ann}\left(D / C_{1}\right) .
$$

We show that

$$
J_{1} \subseteq I \quad \text { or } \quad J_{2} \subseteq I .
$$

Seeking a contradiction, we suppose that both inclusions fail. Since $I$ is a maximal ideal, we get $I+J_{1}=N$ and $I+J_{2}=N$. Thus there are $\boldsymbol{i}_{1}, \boldsymbol{i}_{2} \in I$ and $\boldsymbol{j}_{1} \in J_{1}, \boldsymbol{j}_{2} \in J_{2}$ with

$$
\boldsymbol{i}_{1}+\boldsymbol{j}_{1}=\mathrm{id} \quad \text { and } \boldsymbol{i}_{2}+\boldsymbol{j}_{2}=\mathrm{id} .
$$

We observe that $\boldsymbol{j}_{1} \circ \boldsymbol{j}_{2}$ lies in $\operatorname{Ann}(D / C)$ because $\boldsymbol{j}_{1} \cdot\left(\boldsymbol{j}_{2} \cdot D\right) \subseteq \boldsymbol{j}_{1} \cdot C_{1} \subseteq C$. By the assumption, $\boldsymbol{j}_{1} \circ \boldsymbol{j}_{2}$ therefore lies in $I$. By (1.6), $\boldsymbol{j}_{1}$ and $\boldsymbol{j}_{2}$ are both congruent to id modulo $I$. This implies id $\circ$ id $\in I$, yielding the contradiction id $\in I$, and thus completing the proof of (1.5). Since every chain in Id $\Gamma$ is finite, repeating this process allows us to obtain $C^{\prime}, D^{\prime} \in \operatorname{Id} \Gamma$ with $C \leqslant C^{\prime} \prec D^{\prime} \leqslant D$ and

$$
\operatorname{Ann}\left(D^{\prime} / C^{\prime}\right) \leqslant I
$$

Since $C^{\prime} \prec D^{\prime}, N / \operatorname{Ann}\left(D^{\prime} / C^{\prime}\right)$ is 2-primitive with DCCL, and thus simple, and so $\operatorname{Ann}\left(D^{\prime} / C^{\prime}\right)$ is a maximal ideal of $N$, and therefore equal to $I$. Since $N$ has the DCCL, Corollary 1.4 yields that the $N$-groups $D^{\prime} / C^{\prime}$ and $B / A$ are $N$-isomorphic.

We can now resume the proof of Theorem 1.1 suspended on p. 381 . 
Continuation of the proof of Theorem 1.1. Having proved (1.4), we can use Lemma 1.5 to produce ideals $0 \leqslant C_{1} \prec C_{2} \leqslant H$ such that $C_{2} / C_{1}$ and $D / D^{-}$are $N$-isomorphic. We take $A$ to be minimal in Id $\Gamma$ with $A \leqslant C_{2}, A \nless C_{1}$. Then $A$ is join irreducible and the interval $I\left[A^{-}, A\right]$ projects up to $I\left[C_{1}, C_{2}\right]$ in Id $\Gamma$. Thus, by Lemma 1.2, $A / A^{-}$and $C_{2} / C_{1}$ are $N$-isomorphic. Since $A \leqslant C_{2} \leqslant H, A$ lies in $I[0, H]$. Every ideal in $I[0, H]$ does not lie in $\mathcal{B}$ (take $\boldsymbol{p}:=$ id in (1.1)), and since $D \in \mathcal{B}$, we get $D \nless H$. So $A$ and $D$ are two join-irreducible ideals of $\Gamma$ such that $A / A^{-}$and $D / D^{-}$are $N$-isomorphic, $A \leqslant H$ and $D \nless H$, contradicting the assumptions. This completes the proof of (2) $\Rightarrow(1)$ of Theorem 1.1.

\section{Polynomials on a direct product without skew congruences}

We shall now investigate the polynomial functions on an $\Omega$-group $V$ which is a direct product of similar (cf. [10, p. 13]) $\Omega$-groups; we do so by applying Theorem 1.1 to the near-ring $P_{0}(V)$ of zero-preserving polynomial functions on $V$. For the notion of $\Omega$-group, we refer to [7]; polynomial functions are defined, for example, in [9] or [10, Definition 4.4]. The near-ring operations of $P_{0}(V)$ are pointwise addition of functions, and functional composition. If $V$ is a group (without further operations), then this near-ring is more commonly known as the inner automorphism near-ring $I(V)$.

Let $R_{1}$ and $R_{2}$ be two commutative rings with unit. It is well known that $P_{0}\left(R_{1} \times R_{2}\right)$ is isomorphic to $P_{0}\left(R_{1}\right) \times P_{0}\left(R_{2}\right)$ via a (natural) isomorphism. The same is not true for groups: let $G_{1}=G_{2}=\mathbb{Z}_{2}$. We know that $\left|I\left(\mathbb{Z}_{2} \times \mathbb{Z}_{2}\right)\right|=\left|I\left(\mathbb{Z}_{2}\right)\right|=2$, making the desired isomorphism impossible. However, using Scott's concept of length [15], it is possible to show that for finite groups $G_{1}, G_{2}$ we have $I\left(G_{1} \times G_{2}\right) \cong I\left(G_{1}\right) \times I\left(G_{2}\right)$ if and only if $G_{1} \times G_{2}$ has no skew congruences. This is the case if and only if every normal subgroup $N$ of $G_{1} \times G_{2}$ is equal to $N_{1} \times N_{2}$ for some normal subgroups $N_{1}$ of $G_{1}$ and $N_{2}$ of $G_{2}$. The following theorem tells that all $\Omega$-groups behave like groups.

Theorem 2.1. Let $V_{1}, V_{2}, \ldots, V_{k}$ be similar finite $\Omega$-groups, and let $V:=\prod_{i=1}^{k} V_{i}$. Then the following statements are equivalent.

(1) For every ideal $I$ of $V$ there are ideals $I_{1}$ of $V_{1}, I_{2}$ of $V_{2}, \ldots, I_{k}$ of $V_{k}$ such that $I=\prod_{j=1}^{k} I_{j}$.

(2) For every $j \in\{1,2, \ldots, k\}$, the function

$$
\boldsymbol{e}_{j}: V \rightarrow V, \boldsymbol{e}_{j}\left(v_{1}, \ldots, v_{k}\right)=\left(0, \ldots, 0, v_{j}, 0, \ldots, 0\right)
$$

(with $v_{j}$ standing on the $j$ th place) is in $P_{0}(V)$.

Proof. Let $W_{j}$ be the ideal $0 \times \cdots \times 0 \times V_{j} \times 0 \times \cdots \times 0\left(V_{j}\right.$ on the $j$ th place $)$, i.e. $W_{j}$ is the intersection of the kernels of the projections to all but the $j$ th component. Condition (1) is equivalent to

$$
\text { For every ideal } I \text { of } V: \quad I \leqslant \bigvee_{j=1}^{k}\left(I \wedge W_{j}\right)
$$


We first prove $(2) \Rightarrow(1)$ : we show that $(2.1)$ holds. To this end, let $i \in I$. Since $i=\sum_{j=1}^{k} \boldsymbol{e}_{j}(i)$ and $\boldsymbol{e}_{j}(i)$ lies in both $W_{j}$ and $I, i$ also lies in $\bigvee_{j=1}^{k}\left(I \wedge W_{j}\right)$.

$(1) \Rightarrow(2)$. We have to show that $\boldsymbol{e}_{j}$ is in $P_{0}(V)$. For simplicity we assume that $j=1$. Let $W_{1}:=V_{1} \times 0 \times \cdots \times 0$, and let $K_{1}:=0 \times V_{2} \times V_{3} \times \cdots \times V_{k}$. We first construct a polynomial function $\boldsymbol{e}$ with $\boldsymbol{e}(V) \subseteq W_{1}$ and $\boldsymbol{e}\left(w_{1}\right)=w_{1}$ for all $w_{1} \in W_{1}$. By Theorem 1.1, there is such an $e \in P_{0}(V)$ if for all join irreducible ideals $A, D$ of $V$ such that $A / A^{-}$ and $D / D^{-}$are $P_{0}(V)$-isomorphic and $A \leqslant W_{1}$, the ideal $D$ satisfies

$$
D \leqslant W_{1} \text {. }
$$

Suppose $D \notin W_{1}$. By the fact that $V$ has no skew congruences, we know that

$$
D=\left(D \wedge W_{1}\right) \vee\left(D \wedge K_{1}\right)
$$

Since $D \notin W_{1}$, we have $D \wedge W_{1}<D$. But $D$ is join irreducible, and thus we get $D=D \wedge K_{1}$, which implies $D \leqslant K_{1}$. Let $\bar{\delta}$ be the $P_{0}(V)$-isomorphism from $A / A^{-}$to $D / D^{-}$. Then we can define a mapping $\delta$ from $A$ to $D$ such that $\delta(a)+D^{-}=\bar{\delta}\left(a+A^{-}\right)$ for all $a \in A$. We have

$$
\boldsymbol{p}(\delta(a)) \equiv \delta(\boldsymbol{p}(a))\left(\bmod D^{-}\right) \quad \text { for all } \boldsymbol{p} \in P_{0}(V), a \in A,
$$

and also

$$
\delta\left(a_{1}+a_{2}\right) \equiv \delta\left(a_{1}\right)+\delta\left(a_{2}\right)\left(\bmod D^{-}\right) \quad \text { for all } a_{1}, a_{2} \in A .
$$

We define a subset $S$ of $V$ by

$$
S:=\left\{a+\delta(a)+a_{1}+d_{1} \mid a \in A, a_{1} \in A^{-}, d_{1} \in D^{-}\right\} .
$$

We will now prove that $S$ is an ideal of $V$. To this end, we check that for $s_{1}, s_{2} \in S$ and $\boldsymbol{p} \in P_{0}(V)$, we have $s_{1}+s_{2} \in S$ and $\boldsymbol{p}\left(s_{1}\right) \in S$. Let $a+\delta(a)+a_{1}+d_{1}$ and $a^{\prime}+\delta\left(a^{\prime}\right)+a_{1}^{\prime}+d_{1}^{\prime}$ $\left(a, a^{\prime} \in A, a_{1}, a_{1}^{\prime} \in A^{-}, d_{1}, d_{1}^{\prime} \in D^{-}\right)$be elements of $S$. We know that

$$
a+\delta(a)+a_{1}+d_{1}+a^{\prime}+\delta\left(a^{\prime}\right)+a_{1}^{\prime}+d_{1}^{\prime}
$$

is congruent to $a+\delta(a)+a^{\prime}+\delta\left(a^{\prime}\right)$ modulo $A^{-} \vee D^{-}$. Since $A \wedge D=0$, and since for all ideals $I, J$ of $V$ and $i \in I, j \in J$, we have $i+j \equiv j+i(\bmod I \wedge J)$, this is equal to $a+a^{\prime}+\delta(a)+\delta\left(a^{\prime}\right)$, which is congruent to $a+a^{\prime}+\delta\left(a+a^{\prime}\right)$ modulo $D^{-}$. These congruences allow us to write expression (2.3) as $a+a^{\prime}+\delta\left(a+a^{\prime}\right)+e$, where $e \in A^{-} \vee D^{-}$, which proves that (2.3) lies in $S$; thus $S$ is closed under + . For computing

$$
\boldsymbol{p}\left(a+\delta(a)+a_{1}+d_{1}\right),
$$

we observe that this is congruent to $\boldsymbol{p}(a+\delta(a))$ modulo $A^{-} \vee D^{-}$. Since $A \wedge D=0$, we have $\boldsymbol{p}(a+\delta(a))=\boldsymbol{p}(a)+\boldsymbol{p}(\delta(a))$, which is congruent to $\boldsymbol{p}(a)+\delta(\boldsymbol{p}(a))$ modulo $D^{-}$. So we can write $\boldsymbol{p}\left(a+\delta(a)+a_{1}+d_{1}\right)$ as $\boldsymbol{p}(a)+\delta(\boldsymbol{p}(a))+e^{\prime}$, where $e^{\prime}$ is in $A^{-} \vee D^{-}$, which proves that $S$ is closed under the application of zero-preserving unary polynomial functions. By [13, Theorem 7.123], $S$ is an ideal of $V$. 
We compute $S \wedge K_{1}$. Let $s=a+\delta(a)+a_{1}+d_{1}\left(a \in A, a_{1} \in A^{-}, d_{1} \in D^{-}\right)$be an element of $S \wedge K_{1}$. Since $s, \delta(a)$ and $d_{1}$ lie in $K_{1}$, we obtain $a+a_{1} \in K_{1}$. Since $a$ and $a_{1}$ are elements of $W_{1}$, we have $a+a_{1} \in K_{1} \wedge W_{1}=0$. Thus $a \in A^{-}$, and therefore $\delta(a) \in D^{-}$. So all four summands $a, \delta(a), a_{1}, d_{1}$ lie in $A^{-} \vee D^{-}$, which implies

$$
S \wedge K_{1} \leqslant A^{-} \vee D^{-} \text {. }
$$

Now we compute $S \wedge W_{1}$. We assume $a+\delta(a)+a_{1}+d_{1} \in W_{1}$. Since $a$ and $a_{1}$ are in $A$, which is less than or equal to $W_{1}$, we obtain $\delta(a)+d_{1} \in W_{1}$. Thus $\delta(a)+d_{1} \in W_{1} \wedge K_{1}=0$, which implies $\delta(a) \in D^{-}$. Since $\bar{\delta}$ is an isomorphism, we get $a \in A^{-}$. Again, we see that all four summands are in $A^{-} \vee D^{-}$, and so we get

$$
S \wedge W_{1} \leqslant A^{-} \vee D^{-} .
$$

By the fact that $V$ has no skew congruences, we have $S=\left(S \wedge W_{1}\right) \vee\left(S \wedge K_{1}\right)$, and therefore $S \leqslant A^{-} \vee D^{-}$. We will infer the contradiction $A \leqslant A^{-}$from this fact. We fix $a \in A$. Since $a+\delta(a) \in A^{-} \vee D^{-}$, we get $a \in\left(A^{-} \vee D^{-}\right) \vee D=A^{-} \vee D$. Thus, we have $A \leqslant A^{-} \vee D$. We know that the lattice of ideals of $V$ is modular; by this fact and $D \leqslant K_{1}$, we have $A \leqslant\left(A^{-} \vee D\right) \wedge W_{1}=A^{-} \vee\left(D \wedge W_{1}\right)=A^{-} \vee 0=A^{-}$, a contradiction. This proves (2.2). So, by Theorem 1.1, there is an $\boldsymbol{e} \in P_{0}(V)$ with $\boldsymbol{e}\left(w_{1}\right)=w_{1}$ for all $w_{1} \in W_{1}$ and $\boldsymbol{e}(V) \subseteq W_{1}$. What remains for us to determine is the behaviour of $\boldsymbol{e}$ on $V-W_{1}$. To this end, we compute $\boldsymbol{e}\left(w_{1}+k_{1}\right)$ with $w_{1} \in W_{1}, k_{1} \in K_{1}$. The difference $\boldsymbol{e}\left(w_{1}+k_{1}\right)-\boldsymbol{e}\left(w_{1}\right)$ lies in $K_{1}$, and since $\boldsymbol{e}(V) \subseteq W_{1}$, it also lies in $W_{1}$. From $W_{1} \wedge K_{1}=0$, we obtain

$$
\boldsymbol{e}\left(w_{1}+k_{1}\right)=\boldsymbol{e}\left(w_{1}\right)=w_{1} .
$$

Thus $\boldsymbol{e}$ is the required polynomial $\boldsymbol{e}_{1}$.

We will now study the polynomials on a direct product without skew-congruences. By $\mathrm{Pol}_{n} V$, we denote all $n$-ary polynomial functions from $V^{n}$ to $V$.

Corollary 2.2. Let $V_{1}, \ldots, V_{k}$ be similar finite $\Omega$-groups such that $V=\prod_{j=1}^{k} V_{j}$ has no skew-congruences. Then there is a bijective mapping

$$
\Phi: \mathrm{Pol}_{n} \prod_{j=1}^{k} V_{j} \rightarrow \prod_{j=1}^{k} \operatorname{Pol}_{n} V_{j} .
$$

Proof. Let $\boldsymbol{e}_{1}, \ldots, \boldsymbol{e}_{k}$ be the functions in $P_{0}(V)$ produced by Theorem 2.1. We observe that $V_{i}$ is isomorphic to $V / \beta_{i}$, where $\beta_{i}$ is the congruence corresponding to $V_{1} \times \cdots \times$ $V_{i-1} \times 0 \times V_{i+1} \times \cdots \times V_{k}$. If $\alpha$ is a congruence of $V$ and $v \in V$, we write $v / \alpha$ for the congruence class of $v$ modulo $\alpha$. For a polynomial $\boldsymbol{p} \in \operatorname{Pol}_{n} V$ and a congruence $\alpha$ of $V$, $[p]_{\alpha}$ denotes the function in $\operatorname{Pol}_{n} V / \alpha$ defined by $[\boldsymbol{p}]_{\alpha}\left(v_{1} / \alpha, \ldots, v_{n} / \alpha\right):=\boldsymbol{p}\left(v_{1}, \ldots, v_{n}\right) / \alpha$. We define $\Phi$ by

$$
\begin{aligned}
\Phi: \mathrm{Pol}_{n} V & \rightarrow \prod_{i=1}^{k} \operatorname{Pol}_{n} V / \beta_{i} \\
\boldsymbol{p} & \mapsto\left([\boldsymbol{p}]_{\beta_{1}}, \ldots,[\boldsymbol{p}]_{\beta_{k}}\right) .
\end{aligned}
$$


Since $\beta_{1} \wedge \cdots \wedge \beta_{k}$ is the equality relation on $V$, the mapping $\Phi$ is injective. In order to show that $\Phi$ is surjective, we fix

$$
\left(\boldsymbol{q}_{1}, \ldots, \boldsymbol{q}_{k}\right) \in \prod_{i=1}^{k} \operatorname{Pol}_{n} V / \beta_{i} .
$$

Let $\boldsymbol{p}_{1}, \ldots, \boldsymbol{p}_{k} \in \operatorname{Pol}_{n} V$ be such that $\left[\boldsymbol{p}_{i}\right]_{\beta_{i}}=\boldsymbol{q}_{i}$. We observe that $\left[\boldsymbol{e}_{i}\right]_{\beta_{j}}=[\mathbf{0}]_{\beta_{j}}$ for $i \neq j$, whereas $\left[\boldsymbol{e}_{i}\right]_{\beta_{i}}=[\mathbf{i d}]_{\beta_{i}}$. We define

$$
\boldsymbol{p}:=\sum_{i=1}^{k}\left(\boldsymbol{e}_{i} \circ \boldsymbol{p}_{i}\right) .
$$

Then for $i \in\{1, \ldots, k\}$, we obtain

$$
[\boldsymbol{p}]_{\beta_{i}}=\left[\sum_{j=1}^{k} \boldsymbol{e}_{j} \circ \boldsymbol{p}_{j}\right]_{\beta_{i}} .
$$

For every congruence $\alpha$ of $V$, the mapping $\boldsymbol{p} \mapsto[\boldsymbol{p}]_{\alpha}$ is a homomorphism with respect to + and $\circ$, and thus we get

$$
\begin{aligned}
{\left[\boldsymbol{p}_{\beta_{i}}\right.} & =\sum_{j=1}^{k}\left[\boldsymbol{e}_{j}\right]_{\beta_{i}} \circ\left[\boldsymbol{p}_{j}\right]_{\beta_{i}} \\
& =\left[\boldsymbol{e}_{i}\right]_{\beta_{i}} \circ\left[\boldsymbol{p}_{i}\right]_{\beta_{i}} \\
& =[\mathbf{i d}]_{\beta_{i}} \circ\left[\boldsymbol{p}_{i}\right]_{\beta_{i}} \\
& =\left[\boldsymbol{p}_{i}\right]_{\beta_{i}} \\
& =\boldsymbol{q}_{i} .
\end{aligned}
$$

This proves that $\Phi$ is indeed surjective.

\section{Remarks}

For every $\Omega$-group $W$, the set of mappings $\operatorname{Pol}_{n} W$ is closed under the pointwise application of the operations of $W$. To these pointwise operations, we add the $n+1$-ary operation $\circ$ of composition defined by

$$
\boldsymbol{f}_{0} \circ\left(\boldsymbol{f}_{1}, \ldots, \boldsymbol{f}_{n}\right)\left(w_{1}, \ldots, w_{n}\right):=\boldsymbol{f}_{0}\left(\boldsymbol{f}_{1}\left(w_{1}, \ldots, w_{n}\right), \ldots, \boldsymbol{f}_{n}\left(w_{1}, \ldots, w_{n}\right)\right) .
$$

Under the assumptions of Corollary 2.2, the bijective mapping $\Phi$ is then even an isomorphism with respect to these operations.

For the case of groups (without further operations), Theorem 2.1 can also be derived from Scott's results about polynomial functions on groups in [15]. In contrast to the methods applied in the present paper, Scott makes use of the term representation of a polynomial function, and uses this term representation to define a concept of 'degree' for polynomial functions on groups, called length. The concept of length was generalized to $\Omega$-groups in [4], but we do not see how to derive Theorem 2.1 from their results. 
Acknowledgements. In [5], many connections between the congruence lattice of an algebra and its polynomial functions have been established. The join-irreducible elements of the congruence lattice have been used intensively in [6]. The author thanks Pawel M. Idziak for discussions on $[\mathbf{5}, \mathbf{6}]$ during the joint work on $[\mathbf{2}]$. The present paper was supported by project P-12911-INF of the Austrian Science Fund (FWF), Computing with near-rings - efficient algorithms and theoretical investigations.

\section{References}

1. E. Aichinger, On maximal ideals of tame near-rings, Riv. Mat. Univ. Parma (6) 2* (1999), 215-233.

2. E. Aichinger And P. M. IdZiak, Polynomial interpolation in expanded groups, in preparation.

3. G. Betsch, Some structure theorems on 2-primitive near-rings, Colloq. Math. Soc. János Bolyai 6 (1973), 73-102.

4. G. Eigenthaler and J. Wiesenbauer, On the concept of length in the sense of LauschNöbauer and its generalizations, J. Aust. Math. Soc. A 24 (1977), 162-169.

5. D. Hobby and R. McKenzie, The structure of finite algebras, in Contemporary mathematics, vol. 76 (American Mathematical Society, 1988).

6. P. M. Idziak And K. SŁomczyŃska, Polynomially rich algebras, J. Pure Appl. Algebra, in press.

7. A. G. Kurosh, Lectures on general algebra (Chelsea, New York, 1965).

8. T. Y. LAM, A first course in noncommutative rings (Springer, New York, 1991).

9. H. Lausch AND W. NÖBAUER, Algebra of polynomials (North-Holland, Amsterdam, 1973).

10. R. N. McKenzie, G. F. McNulty and W. F. Taylor, Algebras, lattices, varieties, vol. I (Wadsworth \& Brooks/Cole Advanced Books and Software, Monterey, CA, 1987).

11. J. D. P. MeLdrum, Near-rings and their links with groups (Pitman, Boston, MA, 1985).

12. J. D. P. Meldrum And A. Oswald, Near-rings of mappings, Proc. R. Soc. Edinb. A 83 (1979), 213-223.

13. G. F. PILZ, Near-rings, 2nd edn (North-Holland, Amsterdam, 1983).

14. L. H. Rowen, Ring theory, vol. I (Academic, San Diego, CA, 1988).

15. S. D. Sсотт, The arithmetic of polynomial maps over a group and the structure of certain permutational polynomial groups, I, Monatsh. Math. 73 (1969), 250-267.

16. S. D. Sсотт, Formation radicals for near-rings, Proc. Lond. Math. Soc. (3) 25 (1972), 441-464.

17. S. D. Scotr, Tame near-rings and $N$-groups, Proc. Edinb. Math. Soc. (2) 23 (1980), 275-296. 